\title{
Nonoperative treatment of traumatic spinal injuries in Tanzania: who is not undergoing surgery and why?
}

\author{
Noah L. Lessing ${ }^{1} \cdot$ Albert Lazaro $^{2} \cdot$ Scott L. Zuckerman ${ }^{3,4} \cdot$ Andreas Leidinger $^{5} \cdot$ Nicephorus Rutabasibwa $^{2}$. \\ Hamisi K. Shabani ${ }^{2} \cdot$ Roger Härtl ${ }^{4}$
}

Received: 7 October 2019 / Revised: 10 April 2020 / Accepted: 14 April 2020 / Published online: 29 April 2020

(c) The Author(s), under exclusive licence to International Spinal Cord Society 2020

\begin{abstract}
Study design Retrospective, cohort study of a prospectively collected database.

Objectives In a cohort of patients with traumatic spine injury (TSI) in Tanzania who did not undergo surgery, we sought to: (1) describe this nonoperative population, (2) compare outcomes to operative patients, and (3) determine predictors of nonoperative treatment.

Setting Tertiary referral hospital.

Methods All patients admitted for TSI over a 33-month period were reviewed. Variables included demographics, fracture morphology, neurologic exam, indication for surgery, length of hospitalization, and mortality. Regression analyses were used to report outcomes and predictors of nonoperative treatment.

Results 270 patients met inclusion criteria, of which 145 were managed nonoperatively. Demographics between groups were similar. The nonoperative group was young (mean $=35.5$ years) and primarily male $(n=125,86 \%)$. Nonoperative patients had 7.39 times the odds of death $(p=0.003)$. Patients with AO type A0/1/2/3 fractures $(p<0.001)$, ASIA E exams $(p=0.016)$, cervical spine injuries $(p=0.005)$, and central cord syndrome $(p=0.016)$ were more commonly managed nonoperatively. One hundred and twenty-four patients $(86 \%)$ had indications for but did not undergo surgery. After multivariate analysis, the only predictor of nonoperative management was sustaining a cervical injury $(p<0.001)$.

Conclusions Eighty-six percent of nonoperative TSI patients had an indication for surgery. Nonoperative management was associated with an increased risk of mortality. Cervical injury was the single independent risk factor for not undergoing surgery. The principle reason for nonoperative management was cost of implants. While a causal relationship between nonoperative management and inferior outcomes cannot be made, efforts should be made to provide surgery when indicated, regardless of a patient's ability to pay.
\end{abstract}

Supplementary information The online version of this article (https:// doi.org/10.1038/s41393-020-0474-y) contains supplementary material, which is available to authorized users.

Roger Härtl

roger@hartlmd.net

1 University of Maryland School of Medicine, Baltimore, MD, USA

2 Department of Neurosurgery, Muhimbili Orthopedic Institute, Dar es Salaam, Tanzania

3 Vanderbilt Department of Neurosurgery, Vanderbilt University Medical Center, Nashville, TN, USA

4 Weill Cornell Brain and Spine Center, New York-Presbyterian Hospital, Weill Cornell Medical College, New York, NY, USA

5 Department of Neurosurgery, Hospital General de Catalunya, Sant Cugat del Vallès, Spain

\section{Introduction}

Traumatic spine injury (TSI), comprising fractures to the spinal column and spinal cord injury (SCI), is a significant cause of morbidity and mortality worldwide. The burden of TSI is especially challenging in low- and middle-income countries (LMICs), where incidence is higher with a concomitant shortage of spine surgeons and spinal implants $[1,2]$. TSI often results in permanent neurologic injury, which sidelines working-aged males who are no longer able to provide income for their families.

Surgery for TSI is offered in a small number of tertiary centers throughout Sub-Saharan Africa and other LMICs. Despite studies showing that nonoperative treatment is associated with worse outcomes [3], operative rates remain low in resource-constrained settings [4,5]. As explained by 
Nwanko and Uche [5] in Nigeria as well as Ametefe and colleagues [6] in Ghana, implant cost is the principle reason surgery cannot be performed. While many authors have described operative treatment of TSI in LMICs [6-10], less is known about those who do not undergo surgery. Prompt surgical intervention can potentially mitigate tragic disability, thus an appraisal of who is not undergoing surgery is necessary to advance spine trauma care in LMICs.

To better quantify the landscape of spinal trauma in LMICs, we sought to study a cohort of nonoperative TSI patients through the following objectives: (1) describe the population of nonoperative patients, (2) compare outcomes of patients managed nonoperatively to those undergoing surgery, and (3) discern risk factors for receiving nonoperative care. Defining the differences between these groups and the predictors that lead to nonoperative management can help guide specific recommendations for improving care of these patients in resource-limited settings.

\section{Methods}

\section{Study design and clinical setting}

A retrospective, cohort study of prospectively collected data at Muhimbili Orthopedic Institute (MOI), a freestanding orthopedic and neurosurgery hospital in Dar es Salaam, Tanzania, was performed. MOI is the primary referral center for the country and contains 120 general ward beds, 16 intensive care unit (ICU) beds, 10 emergency department beds, and 5 operating rooms. X-ray, computed tomography, and magnetic resonance imaging (MRI) are all available on site.

\section{Patient identification and operative decision- making}

All consecutive patients admitted for TSI over a 33-month period (September 2016 to May 2019) were reviewed. Any patient under the age of 14 or with a concomitant brain injury was excluded. During the study period, no official spine trauma protocol was in place, and both the decision to operate and the surgical plan was based on three factors: (1) the patient's ability to tolerate surgery, (2) availability of spinal implants, and (3) the patient's financial resources to pay for spinal implants. Of these reasons, an inability to pay for spinal implants was the driving factor, as described previously [11]. The current study represents an extension of a prior series, and though nonoperative treatment was the focus of the current manuscript, operative cases were included to serve only as a comparison group [11]. Moreover, while implants require payment, certain ICU services were delivered regardless of cost, including: vasopressors, arterial blood gasses, and ventilator treatment. That said, certain laboratory tests and all imaging required payment.

\section{Clinical data collection}

Independent variables included demographics, injury mechanism, and insurance status, categorized as public or private. Patients with public insurance were required to pay for hospital services before receiving them, while private patients were not required to provide payment upfront. Prehospital care was described by distance from injury site to MOI (km), prior outside hospital (OSH) admission (yes/ no), and days from injury to MOI admission. Fracture type was defined using the AO Classification System (A0-4, B1-3, C) [12]. Patients with traumatic disk herniation or central cord syndrome were classified as A0. Central cord syndrome was defined by radiographic and/or clinical presence of a cervical SCI without ongoing compression, which most often occurred after acute trauma in the setting of preexisting spondylosis and a narrowed canal. We acknowledge that the definition of central cord syndrome is controversial, and cases of spondylosis with active compression due to a fracture, disc herniation, ligamentum flavum, or facet arthropathy, were not defined as central cord syndrome in the present study. Only cervical SCI without evidence of an actively compressed spinal cord was considered central cord syndrome. Level of injury was recorded as axial cervical (occiput-C2), subaxial cervical (C3-C7), thoracic (T1-T12), or lumbar (L1-L5). If the injured levels included $\mathrm{C} 7$ and $\mathrm{T} 1$ or $\mathrm{T} 12$ and $\mathrm{L} 1$, the level of injury was classified as cervicothoracic or thoracolumbar, respectively. Instances with multiple spine injuries were classified according to the more severe level. Neurologic status upon admission and discharge was determined according to the International Neurological Classification of Spinal Injury using the American Spinal Injury Association (ASIA) Impairment Scale [13]. Improvement or decline in neurologic status were defined by change in at least one ASIA grade during the hospital stay.

Outcome variables were collected to determine differences in nonoperative vs. operative care. The four outcomes included: (1) neurologic improvement, defined as improvement in ASIA grade compared with stable or decline; (2) length of stay (days); (3) presence of a pressure ulcer during admission; and (4) mortality during admission.

\section{Surgical indication}

Patients in the nonoperative group were retrospectively reviewed to determine whether an indication for surgery was present. An injury was considered to have a surgical indication in one of the two ways: (1) an unstable fracture, classified as AO type $\mathrm{A} 4, \mathrm{~B}$, or $\mathrm{C}$, which required 
stabilization regardless of neurologic exam, or (2) a potentially unstable fracture, classified as AO type A0-A3, with neurologic impairment (ASIA A-D). Given the limited resources, some patients only had $\mathrm{x}$-rays available due to cost; in these cases, a best estimation was made with AP and lateral radiographs. Surgical indication determinations were performed by two individuals: a neurosurgery trainee and fellowship-trained neurosurgeon.

\section{Statistical analysis}

Continuous variables were reported as mean (SD) and median (range). Categorical data were presented as count $(\%)$. Student's $t$ tests were used for comparing parametric continuous data; Mann-Whitney $U$ tests for nonparametric continuous data; chi-square and Wilcoxon rank sum tests for categorical data. Outcomes of nonoperative vs. operative management were compared using multivariate regression controlling for age, gender, insurance status, mechanism of injury, fracture stability (as defined above), injury level, and exam on admission (ASIA grade). Logistic regression was used for neurologic improvement, pressure ulcers, and mortality, while linear regression was used for length of stay. Predictors of nonoperative treatment were determined by univariate followed by multivariate logistic regression, controlling for age, gender, mechanism of injury, and exam on admission (ASIA grade). Any variable $<0.10$ on univariate analysis was entered into the multivariate regression. Due to multiple comparisons in the predictors of nonoperative care model (9), a Bonferroni correction was applied, and results were considered significant if $p<0.006$. Statistics were performed using RStudio, version 1.2.1335.

\section{Results}

\section{Demographics}

A total of 284 patients were registered into the prospectively collected database during the study period. Of these, 14 patients were excluded because they either: died before obtaining spine imaging (4), did not undergo spine imaging (6), were neurologically intact without fracture on imaging (2), or had missing data (2). Of the remaining 270 patients, 145 were treated nonoperatively while 125 underwent surgery.

Demographics between groups were similar (Table 1). The nonoperative group was primarily young males with public insurance. On average, injuries occurred $307 \mathrm{~km}$ away (range 0-1378) and with a 5.9-day delay (range 0-72) before hospital admission. Sixty-one nonoperative patients (42\%) sustained AO type A0/1/2/3 fractures compared with
$26(21 \%)$ operative patients $(p<0.001)$. Type A4/B/C patterns were seen less frequently in the nonoperative group ( $n=84,58 \%$ vs. $n=99,79 \%$ | $p<0.001)$. Craniocervical injuries were more common in the nonoperative group $(n=$ $10,7 \%$ vs. $n=1,1 \% \mid p=0.027)$, as were injuries to the subaxial cervical spine ( $n=62,43 \%$ vs. $n=32,26 \%$ I $p=0.005$ ).

Patients with intact neurologic status (ASIA E) upon admission were more commonly managed nonoperatively ( $n=32,22 \%$ vs. $n=13,10 \%$ | $p=0.016$ ). Patients with ASIA B exams were less frequently treated nonoperatively $(n=16,11 \%$ vs. $\mathrm{n}=28,22 \% \mid p=0.018)$. There were 11 patients $(8 \%)$ in the nonoperative group compared with only 1 patient $(1 \%)$ in the operative group with diagnoses of central cord syndrome $(p=0.016)$. The average time from injury to surgical treatment was 31 days $(\mathrm{SD}=22.6$ days). Surgeries performed in the operative group included: anterior cervical discectomy and fusion $(n=6)$, anterior cervical corpectomy with plate $(n=10)$, posterior cervical laminectomy and fusion with lateral mass screws and rods $(n=$ 17 ), and thoracolumbar posterolateral decompression and fusion with pedicle screws and rods $(n=92)$.

Length of stay was shorter for nonoperative patients (median 23 days, interquartile range $12-36$ vs. median 35 days, interquartile range 23-49 $\mid p<0.001$ ) and the mortality rate was higher $(n=24,17 \%$ vs. $n=5,4 \%$ I $p=$ 0.002). One hundred patients $(69 \%)$ in the nonoperative group with an indication for surgery survived.

\section{Surgical indication}

Twenty-one patients (14\%) with AO type A0-3 injuries and intact neurologic exams were appropriately managed nonoperatively. Based on the aforementioned criteria of determining a surgical indication, the remaining 124 patients (86\%) had an indication for surgery: 40 patients (28\%) based on unstable fractures, and 84 patients (58\%) based on potentially unstable fractures with a neurologic deficit.

\section{Incomplete spinal cord injury (iSCl) without surgery}

Of patients with an indication for surgery who survived, 36 had an iSCI (ASIA B, C, or D) (Supplemental Table 1). Mean age was 36.3 years $(S D=11.4)$. On average, there was a 7.9-day delay from injury to admission $(\mathrm{SD}=11.4)$. Thirteen patients had ASIA B exams, 13 had ASIA C, and 10 had ASIA D. Most patients $(n=12)$ suffered from type A0 injuries, while 8 had type C, and 5 had type A4. Cervical traumatic disk herniation was the most common diagnosis $(n=7)$, followed by cervical listhesis $(n=6)$ and lumbar burst fracture $(n=6)$. Four patients had central cord syndrome, where no active compression was seen. 
Table 1 Demographics.

Nonoperative Operative $p$ value

$N=145 \quad N=125$

Demographics and presentation

Age, years

Mean, (SD)
Median, (range)
Male, $n(\%)$

$\begin{array}{cc}35.5(11.5) & 34.1(11.7) \\ 35(15-67) & 32(8-74) \\ 125(86) & 101(81) \\ 16(11) & 17(14)\end{array}$

0.332

0.237

0.301

Private insurance, $n(\%)$

m)

Injury site to MOI distance $(\mathrm{km})$

$\begin{array}{llll}\text { Mean, (SD) } & 307(314) & 340(325) & 0.404 \\ \text { Median, (range) } & 195(0-1378) & 273(0-1166) & 0.597 \\ \text { Prior OSH admission, } n(\%) & 140(97) & 117(94) & 0.398\end{array}$

Days from injury to MOI admission

$\begin{array}{lrcc}\text { Mean, (SD) } & 5.9(10.2) & 5.1(11.0) & 0.560 \\ \text { Median, (range) } & 2(0-72) & 2(0-105) & 0.673 \\ \text { maging, } n(\%) & & & \\ \text { X-ray } & 120(83) & 86(69) & 0.011^{*} \\ \text { CT } & 41(28) & 43(34) & 0.341 \\ \text { MRI } & 96(66) & 90(72) & 0.234\end{array}$

Injury characteristics

Mechanism, $n(\%)$

Motor vehicle accident

Motorcycle

$33(23)$

$37(30)$

29 (20)

Pedestrian

$11(8)$

Fall $>3 \mathrm{~m}$

$30(21)$

$15(12)$

$10(8)$

$34(27)$

Fall $<3 \mathrm{~m}$

19 (13)

$6(5)$

Blunt object

Other

AO fracture type, $n(\%)$

$\mathrm{A} 0-\mathrm{A} 3$

$\mathrm{A} 4, \mathrm{~B}$, or $\mathrm{C}$

$16(11)$

20 (16)

7 (5)

3 (2)

$61(42)$

$26(21)$

$84(58)$

99 (79)

Location, $n(\%)$

Axial cervical spine

Subaxial cervical spine

10 (7)

$62(43)$

1 (1)

$0.027 *$

$0.005^{*}$

2 (1)

32 (26)

Cervicothoracic spine

Thoracic spine

Thoracolumbar

Lumbar

Neurologic status, $n(\%)$

ASIA A

ASIA B

ASIA C

ASIA D

ASIA E

Diagnosis of central cord, $n$

(\%)

AO Class A0-A3 + ASIA

37 (26)

0 (0)

0.501

43 (34)

12 (8)

17 (14)

32 (26)

0.144

0.226

$0.047 *$

$71(49)$

$64(51)$

0.807

16 (11)

28 (22)

$0.018^{*}$

15 (10)

7 (6)

0.231

11 (8)

13 (10)

0.551

32 (22)

13 (10)

$0.016^{*}$

1 (1) $0.016^{*}$

$21(14)$

$0(0)$

$<0.001 *$
Table 1 (continued)

\begin{tabular}{lccc}
\hline & $\begin{array}{l}\text { Nonoperative } \\
N=145\end{array}$ & $\begin{array}{l}\text { Operative } \\
N=125\end{array}$ & $p$ value \\
\hline $\begin{array}{l}\text { Outcomes } \\
\text { Pressure ulcer, } n(\%)\end{array}$ & $26(18)$ & $25(20)$ & 0.799 \\
Neurologic status, $n(\%)^{\mathrm{a}}$ & & & \\
$\quad$ Declined & $4(3)$ & $2(2)$ & 0.684 \\
$\quad$ Stable & $105(87)$ & $96(82)$ & 0.408 \\
$\quad$ Improved & $12(10)$ & $19(16)$ & 0.209 \\
Length of stay & & & \\
$\quad$ Mean, (SD) & $27.7(21.8)$ & $38.5(22.9)$ & $<0.001^{*}$ \\
$\quad$ Median, (range) & $23(1-120)$ & $35(6-190)$ & $<0.001^{*}$ \\
Mortality, $n(\%)$ & $24(17)$ & $5(4)$ & $0.002^{*}$ \\
Completed follow up, $n(\%)^{\mathrm{a}}$ & $24(25)$ & $23(30)$ & 0.559 \\
\hline An
\end{tabular}

An injury was considered surgical in one of the two ways: (1) an unstable fracture defined as A4, B, C requiring stabilization, regardless of neurologic exam, or (2) a potentially unstable fracture A0-A3 with neurologic impairment (ASIA A-D).

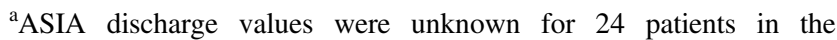
nonoperative group and 8 in the operative group. Follow-up data (yes or no) were missing for 48 patients in the nonoperative group and 44 in the operative group.

*Denotes statistically significant at $p<0.05$.

\section{Mortality}

Twenty-four patients (17\%) in the nonoperative group died, all of whom had indications for surgery (Table 2). Average age was 35.3 years $(\mathrm{SD}=13.1)$. Mean times from injury to admission and admission to death were 1.8 days $(\mathrm{SD}=1.9)$ and 8.1 days $(\mathrm{SD}=6.2)$, respectively. The vast majority of patients $(n=21)$, arrived to the hospital with ASIA A exams. Two patients had ASIA B exams and one patient had an ASIA $\mathrm{C}$ exam. AO type $\mathrm{C}$ injuries were most common $(n=18)$, followed by A0 $(n=3)$ and A2/A4/B1 ( $n=1$ each). Patients primarily suffered from cervical listhesis $(n=12)$. Four patients had cervical listhesis and wedge or burst fractures. The remaining patients had one of the following: atlanto-axial dissociation, cervical burst fracture, cervical spinous process fracture, cervical traumatic disk herniation, cervical central cord syndrome, cervical pincer fracture, thoracic chance fracture, or thoracic listhesis.

\section{Outcomes}

After multivariate analysis controlling for age, gender, insurance status, mechanism of injury, fracture stability, injury level, and exam on admission (ASIA grade), patients managed nonoperatively were 7.39 times more likely to die compared with patients undergoing surgery $(95 \%$ CI: 2.18 , $30.5 \mid p=0.003)$ (Table 3). Patient length of stay when 
Table 2 Nonoperative patients with TSI who did not survive.

\begin{tabular}{|c|c|c|c|c|c|c|}
\hline Case no. & Age, sex & $\begin{array}{l}\text { Injury to } \\
\text { admission (days) }\end{array}$ & $\begin{array}{l}\text { Admission to } \\
\text { death (days) }\end{array}$ & ASIA exam & AO class & Injury \\
\hline 1 & $47, \mathrm{M}$ & 0 & 11 & A & $\mathrm{C}$ & $\begin{array}{l}\text { Atlanto-occipital } \\
\text { dissociation }\end{array}$ \\
\hline 2 & $55, \mathrm{M}$ & 1 & 5 & A & $\mathrm{C}$ & C2 hangman \\
\hline 3 & $45, \mathrm{M}$ & 2 & 2 & $\mathrm{~B}$ & A0 & $\mathrm{C} 3$ spinous process \\
\hline 4 & $43, \mathrm{M}$ & 1 & 1 & A & $\mathrm{C}$ & $\begin{array}{l}\text { C } 3 / 4 \text { listhesis } \\
\text { grade II }\end{array}$ \\
\hline 5 & $21, \mathrm{M}$ & 5 & 4 & A & A4 & $\mathrm{C} 3 / 4$ burst \\
\hline 6 & $43, \mathrm{M}$ & 1 & 7 & A & A0 & $\begin{array}{l}\text { C } 3 / 4 / 5 / 6 \\
\text { central cord }\end{array}$ \\
\hline 7 & $64, \mathrm{M}$ & 2 & 7 & $\mathrm{~B}$ & $\mathrm{~A} 0$ & $\begin{array}{l}\mathrm{C} 3 / 4+\mathrm{C} 6 / 7 \\
\text { traumatic disk } \\
\text { herniations }\end{array}$ \\
\hline 8 & $19, \mathrm{M}$ & 0 & 1 & A & $\mathrm{C}$ & $\begin{array}{l}\mathrm{C} 4 / 5 \text { listhesis } \\
\text { grade } \mathrm{V}+\mathrm{C} 4 \text { burst }\end{array}$ \\
\hline 9 & $37, \mathrm{~F}$ & 0 & 12 & A & $\mathrm{C}$ & C4/5 listhesis I \\
\hline 10 & $20, \mathrm{M}$ & 6 & 12 & A & $\mathrm{C}$ & $\begin{array}{l}\text { C4/5 listhesis II }+ \\
\text { C5 compression }\end{array}$ \\
\hline 11 & $27, \mathrm{M}$ & 5 & 11 & A & $\mathrm{C}$ & C4/5 listhesis I \\
\hline 12 & $18, \mathrm{M}$ & 1 & 6 & A & A2 & C5 pincer \\
\hline 13 & $34, \mathrm{M}$ & 0 & 7 & A & $\mathrm{C}$ & $\begin{array}{l}\text { C5/6 listhesis II + } \\
\text { C5 burst }\end{array}$ \\
\hline 14 & $30, \mathrm{M}$ & 2 & 1 & A & $\mathrm{C}$ & C5/6 listhesis I \\
\hline 15 & $23, \mathrm{~F}$ & 0 & 10 & A & $\mathrm{C}$ & C5/6 listhesis II \\
\hline 16 & $15, \mathrm{M}$ & 0 & 18 & A & $\mathrm{C}$ & C5/6 listhesis II \\
\hline 17 & $50, \mathrm{M}$ & 1 & - & A & $\mathrm{C}$ & C5/6 listhesis II \\
\hline 18 & $29, \mathrm{M}$ & 1 & - & $\mathrm{C}$ & $\mathrm{C}$ & C5/6 listhesis II \\
\hline 19 & $22, \mathrm{~F}$ & 6 & 3 & A & $\mathrm{C}$ & $\begin{array}{l}\text { C6/7 listhesis I + } \\
\text { C6 burst }\end{array}$ \\
\hline 20 & $35, \mathrm{M}$ & 1 & 15 & A & $\mathrm{C}$ & C6/7 listhesis II \\
\hline 21 & $47, \mathrm{M}$ & 2 & 5 & A & $\mathrm{C}$ & C6/7 listhesis I \\
\hline 22 & $36, \mathrm{M}$ & 2 & - & A & $\mathrm{C}$ & C6/7 listhesis I \\
\hline 23 & $47, \mathrm{M}$ & 1 & 26 & A & B1 & T7 chance \\
\hline 24 & $41, \mathrm{M}$ & 3 & 6 & A & $\mathrm{C}$ & $\mathrm{T} 12 / \mathrm{L} 1$ listhesis \\
\hline
\end{tabular}

treated nonoperatively was 8.43 days shorter (95\% CI: $-14.29,-2.56$ । $p=0.005)$. There were no differences in pressure ulcer incidence or number of patients with neurologic improvement.

\section{Predictors of nonoperative treatment}

Prior admission to $\mathrm{OSH}$, fractures classified as potentially unstable (AO type $\mathrm{A} 0-3$ ) as opposed to unstable (AO type $\mathrm{A} 4, \mathrm{~B}$, or $\mathrm{C}$ ), and injury to the cervical spine were significantly associated with nonoperative treatment in univariate analysis (Table 4). The final multivariate model revealed that patients with cervical spine injury were 3.86 times more likely to be managed nonoperatively (95\% CI: $2.24,6.77 \mid p<$ 0.001). Those who were transferred from an OSH were 4.91 times more likely to receive nonoperative treatment, though
Table 3 Comparing outcomes of operative vs. nonoperative treatment; multivariate regression controlled for age, gender, insurance, mechanism, fracture stability, level, and neurologic exam.

\begin{tabular}{llcl}
\hline & Regression & \multicolumn{2}{l}{ Multivariate regression } \\
\cline { 3 - 4 } Variable & & $\mathrm{OR} / \beta(95 \% \mathrm{CI})$ & $p$ \\
\hline Neurologic improvement & Logistic (OR) & $0.50(0.21,1.18)$ & 0.119 \\
Length of stay & Linear $(\beta)$ & $-8.43(-14.29,-2.56)$ & $0.005^{*}$ \\
Pressure ulcers & Logistic (OR) & $1.12(0.55,2.32)$ & 0.748 \\
Mortality & Logistic (OR) & $7.39(2.18,30.46)$ & $0.003^{*}$
\end{tabular}

*Denotes statistically significant at $p<0.05$.

this lost significance $(95 \% \mathrm{CI}: 1.04,35.85 \mid p=0.065)$. Potentially unstable fractures (AO type $\mathrm{A} 0-3$ ) were 1.56 times more likely to be managed nonoperatively, but this lost significance as well (95\% CI: $0.83,2.93 \mid p=0.167)$. 
Table 4 Predictors of receiving nonoperative treatment for TSI among patients with a surgical indication; multivariate logistic regression controlled for age, gender, mechanism, and neurologic exam.

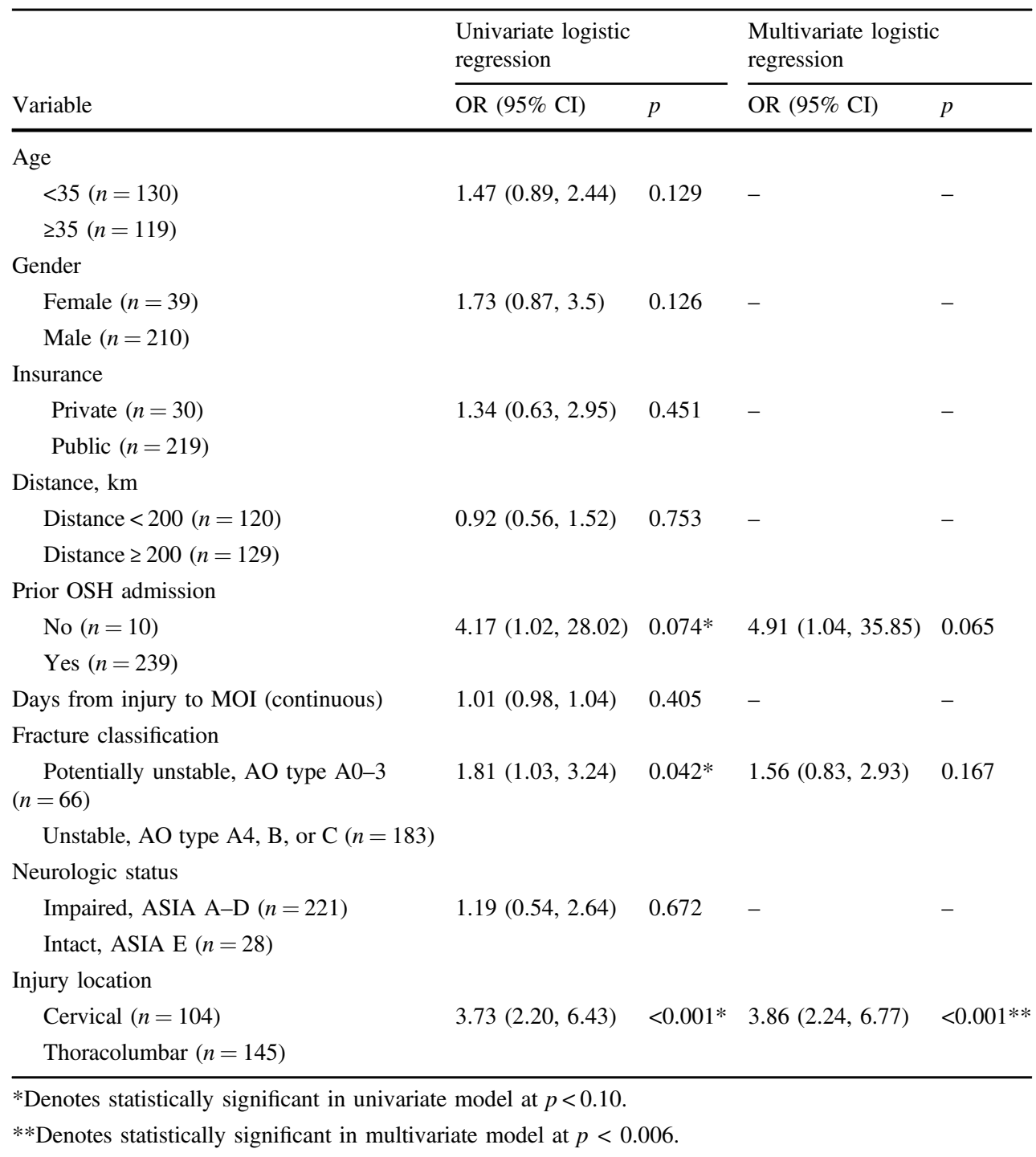

\section{Discussion}

The current study described a large cohort of TSI patients who were managed without surgery at a tertiary referral center in East Africa. Of the 145 patients managed nonoperatively, $86 \%$ had an indication for surgery, and $17 \%$ died $\sim 8$ days post admission. Compared with operative patients, nonoperative patients had seven times the odds of mortality. Injury to the cervical spine was the single independent predictor of nonoperative treatment. Rates of operative treatment for TSI in LMICs vary widely, and the current analysis delves into those who do not undergo surgery, their outcomes, and further areas of study.

Despite the large number of patients with operative indications, several patients with less severe fractures were appropriately managed without surgery. Of the 61 patients with potentially unstable fractures (A0-A3), 34\% were neurologically intact (ASIA E), and thus conservative management was acceptable. Electing to brace patients when possible helps save spinal implants for the most severe injuries and is a judicious use of expensive supplies. Surgery also has its own set of risks. One study from India of 192 TSI patients, 19\% of which underwent surgery, found that surgery was associated with five times the odds of developing a complication, such as pneumonia, urinary tract infection, and reoperation [14]. These results highlight that nonoperative management of less severe fractures without neurologic deficits should be pursued whenever possible.

Outcomes were generally worse for patients who did not undergo surgery. Nonoperative patients had seven times the odds of mortality after multivariate analysis. Given that the average time of death was 8.1 days post admission, these were not polytrauma patients who died shortly after arrival, where little could be done. The high rate of mortality among nonoperative patients is similar to prior studies. In northeast Tanzania, Rashid et al. [4] described a series of 125 TSI patients, $98 \%$ of whom were treated nonoperatively, with a 
mortality rate of $24 \%$. Cervical injuries accounted for $72 \%$ of deaths. Obalum et al. [15] reported an $18 \%$ mortality rate among 468 TSI patients at a Nigerian center; likewise, $72 \%$ of deaths occurred in patients with cervical injuries. Lastly, Moshi et al. [16] in Tanzania found a 24\% mortality rate in 213 TSI patients $-46 \%$ of deaths were from cervical trauma and $10 \%$ of deaths were in patients with central cord syndrome. Unfortunately, there was no mention of whether patients were managed operatively or nonoperatively in the latter two studies. In LMIC centers, not undergoing surgery appears to be tightly associated with increased risk of mortality. Due to the present study design, we cannot definitively say why the increased risk of mortality was seen. One possibility is that patients did not undergo the needed spinal cord decompression in a timely fashion, resulting in compromised neurologic, respiratory, and/or autonomic function, leading to death from downstream causes of the initial SCI. Alternatively, intensive care resources might not have been adequate to support patients with SCI, specifically the need for vasopressors and mechanical ventilation.

The single independent predictor of nonoperative treatment was sustaining a cervical injury, which is consistent with prior series. Choi et al. [9] in Cambodia performed stabilizations for $79 \%$ of thoracic/lumbar injuries compared with $68 \%$ of cervical injuries. Nwankwo and Uche [5] in Nigeria stabilized $19 \%$ of lumbar injuries, but only $9 \%$ of cervical injuries. Ametefe et al. [6] in Ghana treated 52\% of thoracic injuries and $82 \%$ of lumbar injuries, compared with only $38 \%$ of cervical injuries. Though at first glance, this may seem counterintuitive, it is more common for these severe cervical injuries to not be operated on due to late presentation, the severity of neurologic deficits, poor prognosis, and complexity of the indicated surgery. Our finding could represent several phenomena. First, a dearth of cervical implants could exist, namely lateral mass screws, cervical cages, and most importantly set screws, which anecdotally appear to be the scarcest resource. The same can be said for finer instruments needed for anterior approaches, such as small, sharp curettes, and kerrisons $[6,17]$. Second, high cervical injuries are more complex, with critical structures such as the vertebral artery, lower brainstem, and cranial nerves at play. Additional training may be required for these procedures, especially craniocervical surgery, as only 1 of 11 patients with craniocervical injuries underwent surgery in the current series. Third, there was a trend toward nonoperative management of central cord syndrome. Despite evidence that these patients benefit from early decompression [18, 19], treatment for central cord syndrome is still debated, and nonoperative care is sometimes preferred [20], which likely explains these results. Decompressing patients with central cord syndrome may be a reasonable option to pursue, especially in absence of fracture or instability, as a laminectomy could be all that is needed.

The principle reason why over two-thirds of patients did not undergo surgery despite having indications was cost-a theme alluded to previously. Nwankwo and Uche [5] in Nigeria were only able to operate on $57 \%$ of 30 patients with surgical indications because patients were unable to afford implants. Similarly, Ametefe et al. [6] in Ghana mentioned that cost of surgery accounted for operating on only $47 \%$ of 185 patients with TSI. Even worse, in an operative series of 146 TSI patients by Lehre et al. [7] in Ethiopia, neither the patients nor the hospital could afford implants, forcing them to rely entirely on donations. Seeking solutions, some LMIC centers have employed costeffective methods of spinal fixation, such as the Adeolu technique in Nigeria, where stabilization is achieved with a modified Rush nail as a vertical strut secured to the spine with sublaminar cerclage wire [21]. The same authors used this technique in 30 patients with TSI, and $94 \%$ of patients were "very satisfied" or "satisfied" with the procedure $[8,22]$. Wiring for cervical injuries and Luque rods for multilevel thoracic and lumbar fixation have also been used [9]. Interestingly, in a Cambodian series [9], five patients underwent cervical laminoplasty, which is a cost-effective treatment for certain central cord syndrome when only decompression is needed. For patients who can afford implants, the delay to accumulate sufficient funds directly increased length of stay, as reflected in the current series where operative patients remained in the hospital for 9 days longer.

From these results, we offer the following recommendations to make surgery a viable option for all TSI patients in LMICs:

(1) Provide spinal implants, especially cervical. Although donations bolster short-term supply, they are not a sustainable solution. We encourage hospitals to negotiate with vendors for low-cost implants, and governments to restructure insurance programs to cover operative TSI care.

(2) Pursue educational training on cervical surgery. Symposiums and educational conferences are more frequently being held in LMICs. Future demonstrations should emphasize placement of cervical instrumentation, especially at the craniocervical junction.

(3) Emphasize operative treatment for central cord syndrome. The incidence of central cord syndrome will continue to grow with an aging population [23]. Surgeons should be aware of incomplete injuries in older patients without fracture or dislocation, and offer surgical decompression as a treatment option. That said, the hospital should have the needed resources to treat these postoperative patients, which 
can be complex, including ICU care, vasopressors, ventilators, and experienced nursing.

(4) Augment ICU care to support airway and blood pressure management. When possible, ICUs should staff a neuro-intensivist to provide care to TSI patients in the acute setting and be cognizant of the neurologic, respiratory, and/or autonomic dysfunction that will occur.

(5) Provide basic education on spine trauma management to regional hospitals. Nearly all TSI patients are stalled for several days at regional hospitals before being transferred to tertiary centers [24]. Teaching basic physical exam and immobilization techniques would streamline referral to larger institutions for definitive management.

(6) Implement a spine trauma protocol that treats all patients regardless of cost. Eliminating the financial requirement for spinal implants has potential to improve outcomes and decrease lengths of stay.

There are several limitations to the current study. Followup information was not collected, so it is unknown how nonoperative treatment impacted long-term neurologic status and radiographic healing. Also, the results may be difficult to generalize to other neurosurgery and orthopedic departments in LMICs, because each institution has a unique set of available resources that dictate operative decision-making. Several important variables could not be controlled for, including comorbidities and socioeconomic status. Ideally, each patient could be directly asked about their employment status, individual income, family income, and education level; however, this was not feasible, and insurance status was used as a surrogate for socioeconomic status. Socioeconomic factors are especially important because patients with more financial resources can pay for better care, expensive implants, and rehabilitation resources, such as wheelchairs. For these reasons, socioeconomic factors can interact heavily with many outcomes. Regarding comorbidities, although the majority of patients were young males without medical problems, older, sicker patients have decreased physiologic reserve and are less likely to survive a devastating SCI. Comorbidities, unfortunately, were not assessed. In addition, determination of central cord syndrome was defined by radiographic or clinical evidence of cervical SCI without ongoing compression and assessed retrospectively. MRI scans were not available on all patients, which means that retrospectively diagnosing patients with central cord syndrome was at times an approximation and educated guess without complete imaging. Finally, determination of surgical indication is a complex process, and doing this retrospectively without access to a detailed patient history and complete imaging, may have led to misclassifications.

\section{Conclusions}

The current study summarizes the nonoperative management of a large cohort of TSI patients from a tertiary referral hospital in Tanzania. Of 270 total patients, 145 were managed without surgery. Nonoperative patients had seven times the increased odds of death and cervical injuries were the single independent predictor of nonoperative care. While causation between nonoperative treatment and inferior outcomes cannot be made, it is possible that many patients would have benefitted from surgery if implants were provided free of charge. Future work should define the cost of TSI surgery in LMICs and devise ways to reduce the financial burden imposed on these patients.

\section{Data availability}

The datasets generated and analyzed during the current study are available from the corresponding author on reasonable request.

Author contributions NLL was responsible for statistical analysis, interpretation of the results, and writing the manuscript. AL was responsible for collection of data, interpretation of the results, and writing the manuscript. SLZ was responsible for conception of study design, interpretation of the results, and editing the manuscript. AL, $\mathrm{NR}$, and HKS were responsible for interpretation of the results and editing the manuscript. RH was responsible for conception of study design, interpretation of the results, editing the manuscript, and supervision.

\section{Compliance with ethical standards}

Conflict of interest The authors declare that they have no conflict of interest.

Ethics approval We certify that all applicable institutional and governmental regulations concerning the ethical use of human volunteers were followed, including institutional review board approval.

Publisher's note Springer Nature remains neutral with regard to jurisdictional claims in published maps and institutional affiliations.

\section{References}

1. GBD Traumatic Brain Injury and Spinal Cord Injury Collaborators. Global, regional, and national burden of traumatic brain injury and spinal cord injury, 1990-2016: a systematic analysis for the Global Burden of Disease Study 2016. Lancet Neurol. 2019;18:56-87.

2. Kumar R, Lim J, Mekary RA, Rattani A, Dewan MC, Sharif SY, et al. Traumatic spinal injury: global epidemiology and worldwide volume. World Neurosurg. 2018;113:e345-63.

3. La Rosa G, Conti A, Cardali S, Cacciola F, Tomasello F. Does early decompression improve neurological outcome of spinal cord injured patients? Appraisal of the literature using a meta-analytical approach. Spinal Cord. 2004;42:503-12. 
4. Rashid SM, Jusabani MA, Mandari FN, Dekker MCJ. The characteristics of traumatic spinal cord injuries at a referral hospital in Northern Tanzania. Spinal Cord Ser Cases. 2017;3:17021.

5. Nwankwo OE, Uche EO. Epidemiological and treatment profiles of spinal cord injury in southeast Nigeria. Spinal Cord. 2013;51: 448-52.

6. Ametefe MK, Bankah PE, Yankey KP, Akoto H, Janney D, Dakurah TK. Spinal cord and spine trauma in a large teaching hospital in Ghana. Spinal Cord. 2016;54:1164-8.

7. Lehre MA, Eriksen LM, Tirsit A, Bekele S, Petros S, Park KB, et al. Outcome in patients undergoing surgery for spinal injury in an Ethiopian hospital. J Neurosurg Spine. 2015;23:772-9.

8. Rabiu TB. Clinical outcomes of posterior spinal stabilization with rigid vertical strut and spinal process wires (the Adeolu's technique) in a developing country. Pan Afr Med J. 2017;26:84.

9. Choi JH, Park PJ, Din V, Sam N, Iv V, Park KB. Epidemiology and clinical management of traumatic spine injuries at a major government hospital in Cambodia. Asian Spine J. 2017;11: 908-16.

10. Ojo OA, Poluyi EO, Owolabi BS, Kanu OO, Popoola MO. Surgical decompression for traumatic spinal cord injury in a tertiary center. Niger J Clin Pr. 2017;20:1455-60.

11. Magogo JLA, Mango M, Zuckerman SL, Leidinger A, Msuya S, Rutabasibwa N, et al. Operative treatment of traumatic spinal injuries in Tanzania: surgical management, neurologic outcomes, and time to surgery. Global Spine J. 2020. https://doi.org/10.1177/ 2192568219894956.

12. Schnake KJ, Schroeder GD, Vaccaro AR, Oner C. AOSpine classification systems (subaxial, thoracolumbar). J Orthop Trauma. 2017;31(Suppl 4):S14-S23.

13. Kirshblum SC, Waring W, Biering-Sorensen F, Burns SP, Johansen M, Schmidt-Read M, et al. Reference for the 2011 revision of the international standards for neurological classification of spinal cord injury. J Spinal Cord Med. 2011;34: 547-54.
14. Aleem IS, DeMarco D, Drew B, Sancheti P, Shetty V, Dhillon M, et al. The burden of spine fractures in india: a prospective multicenter study. Glob Spine J. 2017;7:325-33.

15. Obalum DC, Giwa SO, Adekoya-Cole TO, Enweluzo GO. Profile of spinal injuries in Lagos, Nigeria. Spinal Cord. 2009;47:134-7.

16. Moshi H, Sundelin G, Sahlen KG, Sorlin A. Traumatic spinal cord injury in the north-east Tanzania-describing incidence, etiology and clinical outcomes retrospectively. Glob Health Action. 2017; 10:1355604.

17. Admasu AK, Buno E. Short-term outcome in subaxial spine injury patients operated on in a resource-limited setting, Addis Ababa, Ethiopia. World Neurosurg. 2018;113:e702-6.

18. Lenehan B, Fisher CG, Vaccaro A, Fehlings M, Aarabi B, Dvorak MF. The urgency of surgical decompression in acute central cord injuries with spondylosis and without instability. Spine (Philos $\mathrm{Pa}$ 1976). 2010;35(21 Suppl):S180-S186.

19. Anderson KK, Tetreault L, Shamji MF, Singh A, Vukas RR, Harrop JS, et al. Optimal timing of surgical decompression for acute traumatic central cord syndrome: a systematic review of the literature. Neurosurgery. 2015;77(Suppl 4):S15-S32.

20. Roth EJ, Lawler MH, Yarkony GM. Traumatic central cord syndrome: clinical features and functional outcomes. Arch Phys Med Rehabil. 1990;71:18-23.

21. Adeolu AA, Komolafe EO, Kolawole OA. The technique of using rigid vertical strut and spinal process wire for posterior spinal stabilization. J Spinal Disord Tech. 2011;24:406-8.

22. Adeolu AA, Komolafe EO. Outcome of a posterior spinal fusion technique using spinous process wire and vertical strut. Ann Afr Med. 2014;13:30-34.

23. Thompson C, Mutch J, Parent S, Mac-Thiong JM. The changing demographics of traumatic spinal cord injury: an 11-year study of 831 patients. J Spinal Cord Med. 2015;38:214-23.

24. Leidinger A, Kim EE, Navarro-Ramirez R, Rutabasibwa N, Msuya SR, Askin G, et al. Spinal trauma in Tanzania: current management and outcomes. J Neurosurg Spine. 2019;31:103-11. 